\title{
Anti-obesity efficacy of nanoemulsion oleoresin capsicum in obese rats fed a high-fat diet
}

This article was published in the following Dove Press journal:

International Journal of Nanomedicine

3 January 2014

Number of times this article has been viewed

\author{
Joo-Yeon Kim ${ }^{1, *}$ \\ Mak-Soon Lee ${ }^{1, *}$ \\ Sunyoon Jung' \\ Hyunjin Joo' \\ Chong-Tai Kim² \\ In-Hwan $\mathrm{Kim}^{3}$ \\ Sangjin Seo' \\ Soojung $\mathrm{Oh}^{1}$ \\ Yangha Kim'
}

'Department of Nutritional Science and Food Management, Ewha Womans University, Seoul, Republic of Korea;

${ }^{2}$ Functional Materials Research Group, Korea Food Research Institute,

Seongnam, Gyeonggi, Republic of Korea; ${ }^{3}$ Department of Food and Nutrition, Korea University, Seoul, Republic of Korea

*These authors contributed equally to this work

Correspondence: Yangha Kim Department of Nutritional Science and Food Management, Ewha Womans University, 52 Ewhayeodae-gil, Seodaemun-gu, Seoul I20-750,

Republic of Korea

Tel +82 232774425

Fax +82 232774425

Emailyhmoon@ewha.ac.kr
Purpose: This study determined the effects of oleoresin capsicum (OC) and nanoemulsion OC (NOC) on obesity in obese rats fed a high-fat diet.

Methods: The rats were randomly separated into three groups: a high-fat (HF) diet group, $\mathrm{HF}+\mathrm{OC}$ diet group, and HF + NOC diet group. All groups were fed the diet and water ad libitum for 14 weeks.

Results: NOC reduced the body weight and adipose tissue mass, whereas OC did not. OC and NOC reduced mRNA levels of adipogenic genes, including peroxisome proliferator-activated receptor $(P P A R)-\gamma$, sterol regulatory element-binding protein-1c, and fatty acid-binding protein in white adipose tissue. The mRNA levels of genes related to $\beta$-oxidation or thermogenesis including PPAR- $\alpha$, palmitoyltransferase- $1 \alpha$, and uncoupling protein- 2 were increased by the OC and NOC relative to the HF group. Both OC and NOC clearly stimulated AMP-activated protein kinase (AMPK) activity. In particular, PPAR- $\alpha$, palmitoyltransferase- $1 \alpha$, uncoupling protein-2 expression, and AMPK activity were significantly increased in the NOC group compared to in the OC group. NOC decreased glycerol-3-phosphate dehydrogenase activity whereas $\mathrm{OC}$ did not.

Conclusion: From these results, NOC could be suggested as a potential anti-obesity agent in obese rats fed a HF diet. The effects of the NOC on obesity were associated with changes of multiple gene expression, activation of AMPK, and inhibition of glycerol-3-phosphate dehydrogenase in white adipose tissue.

Keywords: oleoresin capsicum, nanoemulsion, anti-obesity, gene expression, AMP-activated protein kinase, rats

\section{Introduction}

Obesity results from excess energy intake over energy expenditure. Currently, obesity is a major worldwide health problem because of its rising prevalence and strong association with pathological disorders such as heart disease, type 2 diabetes, hypertension, and some forms of cancer. ${ }^{1}$ In the field of food science, studies on obesity have focused on searching for food components that have the potential to suppress the accumulation of body fat and increase energy expenditure. Numerous natural products, including crude extracts and isolated compounds from plants, have been linked to the reduction of body weight and prevention of diet-induced obesity. ${ }^{2}$

Oleoresin capsicum (OC) is an organic solvent extract of dried ripe red pepper, which is the fruit from plants in the genus Capsicum. ${ }^{3} \mathrm{OC}$ is widely used as a food additive for the preservation and enhancement of taste as it has little odor and a pungent taste. The ethanolic and butanolic capsicum extracts present various biological benefits, 
such as anti-cancer, ${ }^{4}$ anti-oxidant, and anti-inflammatory properties. $^{5}$ Moreover, anti-obesity activity of methanol extract from hot pepper (Capcisum annuum L.) seeds has been observed in 3T3-L1 adipocytes with decreased glycerol3-phosphate dehydrogenase (GPDH) activity and CCAATenhancer-binding protein $(C / E B P)-\alpha$ and $C / E B P-\beta$ mRNA expression. ${ }^{6}$ Currently, there are more than 100 compounds identified in OC, such as acids, esters, alcohols, aldehydes, ketones, and carotenoid pigments, as well as capsaicin analogs. ${ }^{7}$ It is assumed that some of those components may exert beneficial effects on health in cooperation with each other; however, the application of OC is limited by some drawbacks, such as low solubility and poor bioavailability, ${ }^{8}$ thus, biotechnologies have been considered to increase bioavailability of OC.

Nanoemulsions (radius $<100 \mathrm{~nm}$ ) are used in the food industry to encapsulate, protect, and deliver lipophilic functional components. ${ }^{9}$ The advantages of nanoemulsion are to improve water solubility, thermal stability, oral bioavailability, sensory attributes, and physiological performance. ${ }^{10}$ Since some bioactive components are hardly soluble in aqueous systems, adding those ingredients to liquid food products is almost impossible or not sufficient for food fortification. ${ }^{11}$ Furthermore, finding appropriate delivery systems is especially challenging for the food industry, compared to other fields such as pharmacy or cosmetics, since only a limited amount of ingredients can be used as encapsulation and stabilization material. ${ }^{11}$ Therefore, the nanoemulsion delivery system available for barely soluble food components could be one solution to enhance health benefits to the consumer.

The aim of the present study was to investigate the effects of OC and nanoemulsion OC (NOC) on obesity in rats with high-fat (HF)-diet-induced obesity. Expression of genes related to adipogenesis, such as peroxisome proliferatoractivated receptor $(P P A R)-\gamma$, sterol regulatory elementbinding protein $(S R E B P)-1 \mathrm{c}$, and fatty acid-binding protein 2 (aP2), were analyzed in white adipose tissue (WAT). The mRNA levels of PPAR- $\alpha$, carnitine palmitoyltransferase $(C P T)-1 \alpha$, and uncoupling protein-2 (UCP2), involved in $\beta$-oxidation and thermogenesis, were evaluated. In addition, activities of AMP-activated protein kinase (AMPK) and GPDH were compared.

\section{Materials and methods \\ Materials}

TRIzol $^{\circledR}$ Reagent and Moloney murine leukemia virus (M-MLV) reverse transcriptase were obtained from Life Technologies (Carlsbad, CA, USA). Universal SYBR ${ }^{\circledR}$ Green
PCR Master Mix was obtained from Qiagen (Germantown, MD, USA). Kits for the analysis of aspartate aminotransferase (AST), alanine aminotransferase (ALT), triglycerides (TGs), and total cholesterol (TC) were purchased from Asan Pharmaceutical (Seoul, Korea). The AMPK Kinase Assay kit was purchased from CycLex (Nagano, Japan). A GPDH activity assay kit was purchased from Takara Bio (Kyoto, Japan). A bicinchoninic acid (BCA) protein assay kit was obtained from Thermo Fisher Scientific (Waltham, MA, USA). All other reagents were obtained from Sigma-Aldrich (St Louis, MO, USA).

\section{Preparation and characterization of OC and NOC}

Samples (OC and NOC) were kindly supplied from Korea Food Research Institute (Songnam, Gyeonggi, Korea). In order to prepare the NOC, a pre-mixture was formulated by stirring $210 \mathrm{~g}$ of OC (General Foods and Flavors, Seoul, Korea) and $201 \mathrm{~g}$ of Tween 80, and the mixture was dispersed into 2,100 $\mathrm{mL}$ of purified water under mild stirring for 1 hour. The coarse emulsion was passed through an air-driven Microfluidizer ${ }^{\circledR}$ (model M-110L; Microfluidics, Westwood, MA, USA) operating at 25,000 psi for five cycles. The microfluidized sample was dispersed into $2,500 \mathrm{~mL}$ of $0.2 \% \beta$-cyclodextrin solution for 2 hours using the syringe pump, and filtered with a $0.45 \mu \mathrm{m}$ polyvinylidene difluoride filter membrane, then stabilized for 24 hours. The transparent solution was considered to be NOC. The control OC sample was prepared by dispersion of OC ( $210 \mathrm{~g})$, surfactant Tween $80(201 \mathrm{~g})$, and 2,100 $\mathrm{mL}$ of purified water. The emulsion was then simply dispersed into $2,500 \mathrm{~mL}$ of $0.2 \% \beta$-cyclodextrin solution without microfluidization. The morphology of nanoemulsions was examined in a transmission electron microscope (Tecnai 12; FEI Company, Eindhoven, the Netherlands). For negative staining, $7 \mu \mathrm{L}$ of the annealed nanoemulsions were placed on carbon-coated copper grids and washed once with ultrapure water. Then, the samples were stained immediately with $7 \mu \mathrm{L}$ of $2 \%$ uranyl acetate in ultrapure water, washed with water again, and then air-dried. Images were acquired at $120 \mathrm{kV}$, and the scales were calibrated using a grating replica $3 \mathrm{~mm}$ grid. A BioScan camera (model 792 Gatan, Inc, Pleasanton, CA, USA), and DigitalMicrograph $^{\mathrm{TM}}$ software (version 3.4; Gatan, Inc, Pleasanton, CA, USA) was used to perform the image capture. The distribution of the NOC particle size was between 20 and $50 \mathrm{~nm}$ in diameter (Figure 1). The amounts of capsaicinoid (capsaicin plus dihydrocapsaicin) in the OC and NOC were quantified by high-performance liquid chromatography 


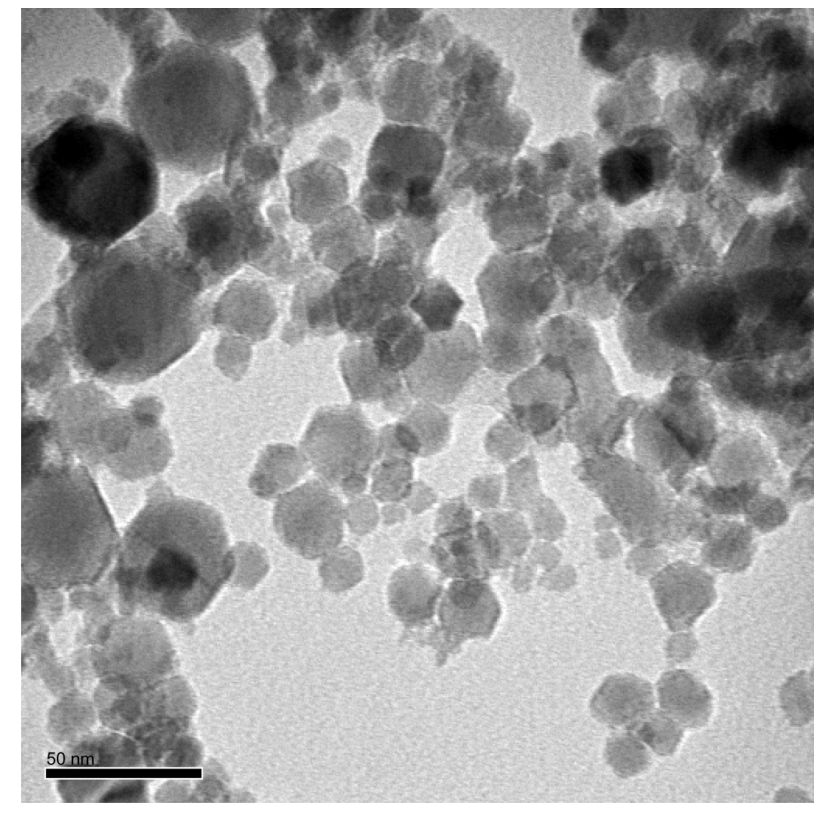

Figure I Transmission electron microscope image of the nanoemulsion oleoresin capsicum (scale bar $=50 \mathrm{~nm}$ ).

using a modified version of a procedure by Davis et al. ${ }^{12}$ Separation was performed on a $\mathrm{C} 18$ column (Zorbax Eclipse XDBC18, $4 \times 6 \times 250 \mathrm{~mm}, 5 \mu \mathrm{m}$; Agilent Technologies, Santa Clara, CA, USA) with a gradient system. The mobile phase was a mixture of methanol and deionized water at a ratio of $70: 30(\mathrm{v} / \mathrm{v})$. The flow rate was $0.8 \mathrm{~mL} /$ minute for 30 minutes, and the ultraviolet/visible detector was set at $280 \mathrm{~nm}$. The amounts of capsaicinoid were $116 \mathrm{mg} / \mathrm{g}$ and $120 \mathrm{mg} / \mathrm{g}$ in the OC and NOC, respectively. The OC and NOC solutions were freeze dried at $-20^{\circ} \mathrm{C}$.

\section{Animals and diets}

Twenty-one male Sprague Dawley rats, 3 weeks of age, were obtained from Daehan Experiment Animals (Eumseong, Korea), and housed individually in stainless steel wire-mesh cages in a room maintained at $22^{\circ} \mathrm{C} \pm 1{ }^{\circ} \mathrm{C}$ with a 12 -hour light/dark cycle (light period: 6 am to $6 \mathrm{pm}$ ). Rats were fed laboratory chow and water ad libitum for 1 week to stabilize their metabolic condition. After the 1-week adaptation, the rats were randomly divided into three groups of seven animals each and subsequently maintained on one of the following diets: a HF control diet (HF group) or a HF control diet supplemented with $0.01 \%$ (wt:wt) OC (OC group), or $0.01 \%$ (wt:wt) NOC (NOC group). The HF control diet ( $45 \%$ of total energy as fat), which contained $23 \%$ (wt:wt) fat, $17 \%$ (wt:wt) casein, 12\% (wt:wt) sucrose, 20\% (wt:wt) starch, 15\% (wt:wt) dextrose, 6\% (wt:wt) cellulose, 4.3\% (wt:wt) minerals, and $1.2 \%$ (wt:wt) vitamins was based on a modification of the AIN-93 diet. ${ }^{13}$ The rats were maintained on these diets for 14 weeks. Body weight and food intake were monitored twice per week. At the end of the experiment, the rats were fasted overnight and anesthetized with Zoletil:Rompun (4:1) (Bayer, Leverkusen, Germany) at a dose of $0.1 \mathrm{~mL} / 80 \mathrm{~g}$ body weight. A central longitudinal incision was made in the abdominal wall, and blood samples were collected by cardiac puncture. Blood samples were centrifuged at $1,500 \times g$ for 20 minutes at $4{ }^{\circ} \mathrm{C}$, and the plasma was separated and stored at $-20^{\circ} \mathrm{C}$ until analyzed. Liver and epididymal and retroperitoneal WAT were harvested, frozen immediately in liquid nitrogen, and stored at $-70^{\circ} \mathrm{C}$. All experimental protocols were approved by the Animal Experimentation Ethics Committee of the Ewha Womans University, Seoul, Korea for the care and use of laboratory animals (permission number: 2009-8-1).

\section{Plasma biochemical measurements}

Plasma levels of AST, ALT, TC, and TG were determined by enzymatic colorimetric methods using commercial kits in accordance with the manufacturer's instructions.

\section{Hepatic and fecal lipids analyses}

Hepatic and fecal lipids were extracted using the method of Bligh and Dyer, ${ }^{14}$ with slight modifications. Briefly, feces and tissues $(500 \mathrm{mg}$ ) were homogenized in $1.5 \mathrm{~mL}$ of $0.9 \%$ saline, and $7.5 \mathrm{~mL}$ of methanol:chloroform $(2: 1, \mathrm{v}: \mathrm{v})$ was added to the homogenates. The mixture was shaken horizontally for 10 minutes and centrifuged at $2,000 \times g$ for 10 minutes. The lower chloroform phase was withdrawn, and the lipid in this phase was dried and weighed. TC and TG concentrations were determined by enzymatic colorimetric methods using commercial kits as described above.

\section{Histological analysis}

Epididymal WAT was dissected from three animals per group and fixed in 10\% (v/v) formalin for 24 hours. The fixed samples were washed and embedded in paraffin. Sections $6 \mu \mathrm{m}$ thick (three sections per rat) were mounted on slides and stained with hematoxylin and eosin stain. The stained sections were observed under a microscope (Olympus, Tokyo, Japan) at $100 \times$ magnification.

\section{Real-time quantitative reverse- transcription polymerase chain reaction}

Total RNA was isolated from WAT using TRIzol ${ }^{\circledR}$ Reagent. The corresponding cDNA was synthesized from $4 \mu \mathrm{g}$ of RNA using M-MLV reverse transcriptase. After cDNA 
synthesis, real-time quantitative reverse-transcription polymerase chain reaction was performed using Universal SYBR $^{\circledR}$ Green PCR Master Mix on a fluorometric thermal cycler (Qiagen, Valencia, CA, USA). The sequences of the sense and antisense primers were designed using an online program. ${ }^{15}$ The sequences of the sense and antisense primers used for amplification are shown in Table 1. The ${ }^{\Delta \Delta} \mathrm{Ct}$ method was used for relative quantification. ${ }^{16}$ The ${ }^{\Delta \Delta} \mathrm{Ct}$ value for each sample was determined by calculating the difference between the $\mathrm{Ct}$ value of the target gene and the $\mathrm{Ct}$ value of the $\beta$-actin reference gene. The normalized level of expression of the target gene in each sample was calculated using the following formula:

$$
2^{-\Delta \Delta \mathrm{Ct}}
$$

Values were expressed as fold change of the HF group.

\section{AMPK activity assay}

AMPK activity was performed as described previously, ${ }^{17}$ using an AMPK Kinase Assay kit in accordance with the manufacturer's instructions. Briefly, samples were incubated for 30 minutes at $30^{\circ} \mathrm{C}$ in a pre-coated plate with a substrate peptide that corresponded to mouse insulin receptor substrates-1 (IRS-1). AMPK activity was measured by monitoring the phosphorylation of Ser 789 in IRS-1 using an anti-mouse phospho-Ser 789 IRS-1 monoclonal antibody and peroxidase-coupled anti-mouse immunoglobulin G. Conversion of the chromogenic substrate tetramethylbenzidine was quantified by measuring the absorbance at $450 \mathrm{~nm}$. Protein was determined using a BCA protein assay kit. Values for AMPK activity were expressed as fold increase of the HF group.

\section{GPDH activity assay}

GPDH activity was measured with a GPDH activity assay kit in accordance with the manufacturer's instructions. Epididymal WAT was homogenized and centrifuged for 10 minutes at $4^{\circ} \mathrm{C}$. GPDH activity was assayed in the supernatant by monitoring the decrease in reduced nicotinamide adenine dinucleotide in the presence of dihydroxyacetone phosphate and measuring absorbance at $340 \mathrm{~nm}$. Protein concentration was determined using a BCA protein assay kit. Values for GPDH activity were expressed as a percentage of the HF group.

\section{Statistical analysis}

All data are expressed as mean \pm standard error of the mean. Statistical analyses were performed using SPSS software (version 17; IBM Corporation, Armonk, NY, USA). Differences among the groups $(\mathrm{HF}, \mathrm{HF}+\mathrm{OC}$, and $\mathrm{HF}+\mathrm{NOC})$ were analyzed by one-way analysis of variance (ANOVA) and Tukey's post hoc multiple comparison tests. $P$-values less than 0.05 were considered to be statistically significant.

\section{Results \\ Body weight, energy intake, and fat accumulation}

At the beginning of the experiment, the initial body weights were not different among the three groups. After 14 weeks of experiment, the final body weight in the NOCsupplemented group was decreased significantly by $10.2 \%$, compared to that in the HF group $(P<0.05)$ (Table 2). The significant reduction in the body weight of the rats supplemented with NOC was shown after 6 weeks of intervention (Figure 2A). The final body weight in the OC-supplemented

Table I Primers used for quantitative real-time polymerase chain reaction (PCR)

\begin{tabular}{|c|c|c|c|c|c|}
\hline Name & GenBank no & Primer sequence $\left(5^{\prime}-3^{\prime}\right)$ & Amplicon size (bp) & Slope & $E(\%)^{a}$ \\
\hline \multirow[t]{2}{*}{$\overline{a P 2}$} & NM_053365 & F: TCACCCCAGATGACAGGAAA & 140 & -3.4928 & 93.3 \\
\hline & & R: CATGACACATTCCACCACCA & & & \\
\hline \multirow[t]{2}{*}{$\beta$-actin } & NM_03II44 & F: GGCACCACACTTTCTACAAT & 123 & -3.3235 & 99.9 \\
\hline & & R: AGGTCTCAAACATGATCTGG & & & \\
\hline \multirow[t]{2}{*}{ CPT-I $\alpha$} & NM_03I559 & F: TCGGCAGACCTATTTTGCAC & 143 & -3.2174 & 104.6 \\
\hline & & R: ATTTGGCGTAGCTGTCGATG & & & \\
\hline \multirow[t]{2}{*}{ PPAR- $\alpha$} & NM_013196 & F: TACCTGTGAACACGATCTGA & 136 & -3.3045 & 100.7 \\
\hline & & R: GCTAGTCTTTCCTGCGAGTA & & & \\
\hline \multirow{2}{*}{ PPAR- $\gamma$} & NM_00II45366 & F: TGTGGGGATAAAGCATCAGC & 175 & -3.4878 & 93.5 \\
\hline & & R: CAAGGCACTTCTGAAACCGA & & & \\
\hline \multirow[t]{2}{*}{ SREBP-IC } & AF286470 & F: AGGAGGCCATCTTGTTGCTT & 134 & -3.2754 & 102.0 \\
\hline & & R: GTTTTGACCCTTAGGGCAGC & & & \\
\hline \multirow[t]{2}{*}{ UCP2 } & NM_019354 & F: ACTGTCGAAGCCTACAAGAC & 111 & -3.4543 & 94.8 \\
\hline & & R: CACCAGCTCAGTACAGTTGA & & & \\
\hline
\end{tabular}

Note: aPCR efficiency $(E)$ was calculated according to the equation $E=\left(10^{[-1 / \text { slope }]}-1\right) \times 100$.

Abbreviations: $a P 2$, fatty acid-binding protein 2; CPT, carnitine palmitoyltransferase; PPAR, peroxisome proliferator-activated receptor; SREBP, sterol regulatory elementbinding protein; UCP2, uncoupling protein-2. 
Table 2 Effects of OC and NOC on physiological variables

\begin{tabular}{|c|c|c|c|}
\hline Variables & HF & HF + OC & HF + NOC \\
\hline Initial body weight (g) & $66.7 \pm 1.3$ & $69.7 \pm 1.9$ & $69.0 \pm 2.5$ \\
\hline Final body weight (g) & $699.9 \pm 15.4$ & $662.1 \pm 8.1$ & $628.6 \pm 22.3 *$ \\
\hline Food intake (g/day) & $25.3 \pm 0.4$ & $23.8 \pm 0.2$ & $23.7 \pm 0.7$ \\
\hline Food efficiency ( $g$ gain/g consumed) & $0.254 \pm 0.005$ & $0.254 \pm 0.003$ & $0.24 I \pm 0.012$ \\
\hline Total food intake (kg//4 weeks) & $2.48 \pm 0.04$ & $2.33 \pm 0.02$ & $2.32 \pm 0.07$ \\
\hline Energy intake (kcal/day) & $117.5 \pm 1.9$ & $110.6 \pm 1.1$ & $109.9 \pm 21.8$ \\
\hline Energy efficiency (g gain/kcal consumed) & $0.055 \pm 0.001$ & $0.055 \pm 0.001$ & $0.052 \pm 0.001$ \\
\hline Total energy intake (kcal//4 weeks) & $11,518.6 \pm 188.9$ & $10,839.7 \pm 105.1$ & $10,776.3 \pm 303.5$ \\
\hline Liver weight (g/l00 g body weight) & $2.9 \pm 0.2$ & $2.9 \pm 0.1$ & $3.1 \pm 0.1$ \\
\hline \multicolumn{4}{|l|}{ Adipose tissue weight ( $\mathrm{g} / \mathrm{I} 00 \mathrm{~g}$ body weight) } \\
\hline Epididymal & $3.34 \pm 0.07$ & $3.58 \pm 0.18$ & $3.1 \pm 0.13^{\#}$ \\
\hline Retroperitoneal & $4.10 \pm 0.26$ & $3.75 \pm 0.12$ & $3.34 \pm 0.17 *$ \\
\hline Total & $7.76 \pm 0.12$ & $7.32 \pm 0.22$ & $6.4 I \pm 0.27 * *, \#$ \\
\hline
\end{tabular}

Notes: Values are expressed as mean \pm standard error of the mean $(n=7)$. $* P<0.05 ; * * P<0.01$ versus $\mathrm{HF}$ group; ${ }^{*} P<0.05$ versus $\mathrm{HF}+\mathrm{OC}$ group.

Abbreviations: HF, high-fat; NOC, nanoemulsion oleoresin capsicum; OC, oleoresin capsicum.

group tended to be lower than that of the HF group, but the difference was not statistically significant throughout the experiment. NOC supplementation significantly reduced the WAT mass by $17 \%$ in comparison with that of the HF group $(P<0.01)$, whereas OC supplementation resulted in no difference in the WAT mass (Table 2). No significant difference in the amount of energy intake and energy efficiency ratio was observed among the three groups over the study period, which indicates that the beneficial effects of the NOC on body weight and the mass of WAT
A

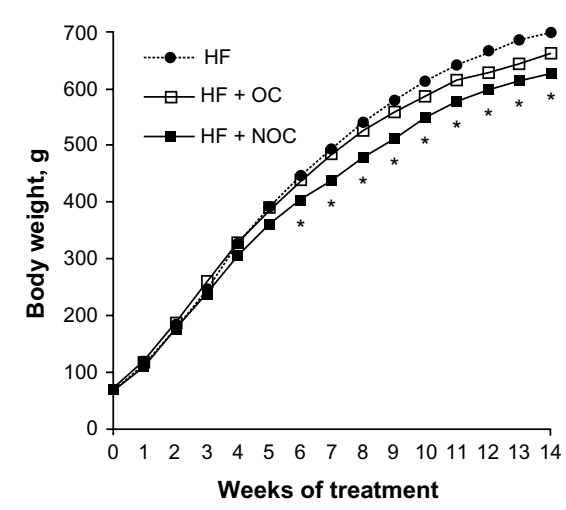

B

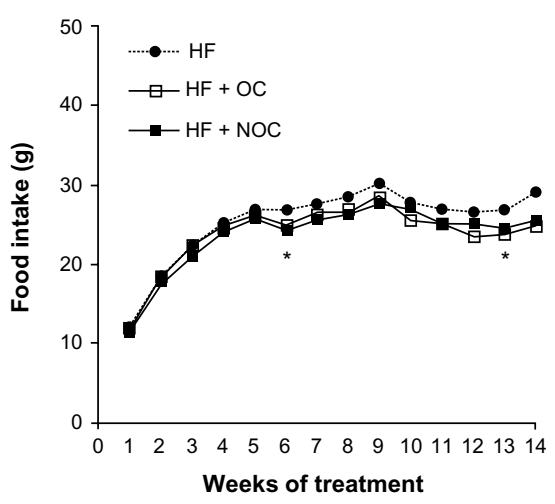

C

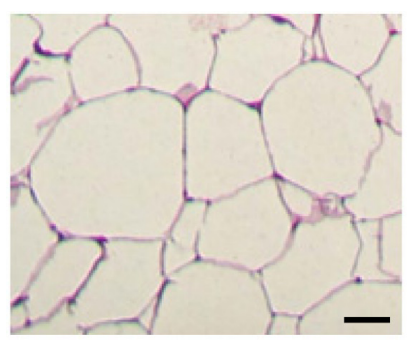

HF

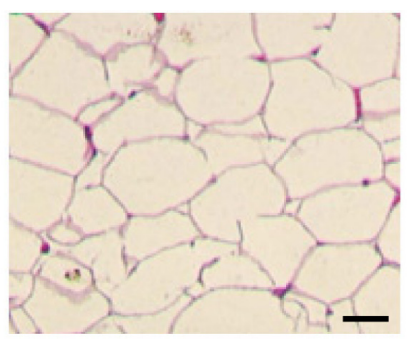

HF + OC

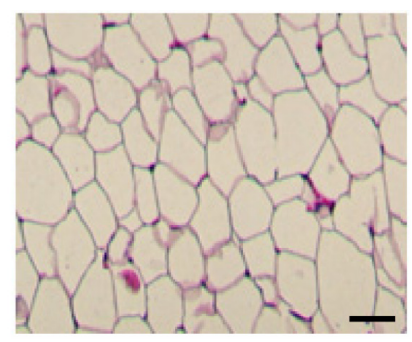

HF + NOC

Figure 2 Effects of $O C$ and NOC on diet-induced obesity.

Notes: Changes in (A) body weight and (B) food intake. (C) Representative histological sections of epididymal adipose tissue (hematoxylin and eosin stain; magnification $100 \times$; scale bar $=100 \mu \mathrm{m})$. Values are expressed as mean \pm standard error of the mean $(n=7)$. $* P<0.05$ versus HF group.

Abbreviations: HF, high-fat; NOC, nanoemulsion oleoresin capsicum; OC, oleoresin capsicum. 
were not caused by reduced energy consumption (Table 2 and Figure 2B).

\section{Plasma, liver, and fecal metabolites}

As shown in Table 3, rats in the NOC-supplemented group showed significantly lower plasma TG concentration by $31 \%$ compared to that of the HF group $(P<0.05)$. However, plasma TG level in the OC-supplemented group was not significantly different compared to that in the HF group. The plasma TC level was not affected by OC and NOC supplementation. Moreover, the levels of TG and TC in the liver and feces were not significantly different among the three groups.

\section{Liver weight and plasma AST and ALT activities}

At the doses given, OC and NOC did not cause a rise in plasma AST and ALT activities when compared to those of the HF group (Table 3 ). In addition, the liver weight was unaffected by the OC and NOC treatment (Table 2). These data indicate that $\mathrm{OC}$ and $\mathrm{NOC}$ were well tolerated by the rats.

\section{Histological analysis}

The WAT histology was performed to compare the effect of OC and NOC on adipocyte size by hematoxylin and eosin staining. The representative histology of epididymal WAT is shown in Figure 2C. The size of epididymal adipocytes was smaller in the OC-and NOC-supplemented groups than in the HF group. In particular, NOC showed a better inhibitory effect on reducing epididymal adipocyte size than OC (Figure 2C).

Table 3 Effects of $\mathrm{OC}$ and NOC on plasma, liver, and fecal metabolites

\begin{tabular}{llll}
\hline Metabolites & HF & HF + OC & HF + NOC \\
\hline Plasma lipids (mmol/L) & & & \\
$\quad$ Triglyceride & $1.33 \pm 0.09$ & $1.17 \pm 0.1 \mathrm{I}$ & $0.91 \pm 0.10^{*}$ \\
$\quad$ Total cholesterol & $1.41 \pm 0.16$ & $1.29 \pm 0.13$ & $0.99 \pm 0.1 \mathrm{I}$ \\
Liver lipids $(\mu \mathrm{mol} / \mathrm{g})$ & & & \\
$\quad$ Triglyceride & $17.5 \pm 1.49$ & $18.9 \pm 3.07$ & $18.9 \pm 2.50$ \\
$\quad$ Total cholesterol & $3.36 \pm 0.17$ & $3.08 \pm 0.24$ & $3.15 \pm 0.16$ \\
Fecal lipids $(\mu \mathrm{mol} / \mathrm{g})$ & & & \\
$\quad$ Triglyceride & $0.80 \pm 0.19$ & $0.57 \pm 0.13$ & $0.53 \pm 0.08$ \\
$\quad$ Total cholesterol & $1.22 \pm 0.23$ & $1.09 \pm 0.29$ & $0.75 \pm 0.15$ \\
Plasma AST (IU/L) & $56.1 \pm 1.6$ & $57.9 \pm 2.6$ & $57.9 \pm 2.5$ \\
Plasma ALT (IU/L) & $21.8 \pm 2.3$ & $23.4 \pm 3.0$ & $25.4 \pm 3.2$ \\
\hline
\end{tabular}

Notes: Values are expressed as mean \pm standard error of the mean $(n=7) . * P<0.05$ versus HF group.

Abbreviations: ALT, alanine aminotransferase; AST, aspartate aminotransferase; HF, high-fat; NOC, nanoemulsion oleoresin capsicum; OC, oleoresin capsicum.
mRNA expression of genes related to adipogenesis, $\beta$-oxidation, and thermogenesis

We next analyzed whether OC or NOC supplementation affected mRNA expression of genes involved in adipogenesis in WAT. The mRNA levels of adipogenic genes such as PPAR- $\gamma, S R E B P-1 c$, and $a P 2$ showed a $60 \%, 33 \%$, and $55 \%$ decrease, respectively, in the OC-supplemented group compared with those of the HF group (Figure 3A). Also, the mRNA levels of PPAR- $\gamma, S R E B P-1 c$, and $a P 2$ showed a $76 \%, 47 \%$, and $79 \%$ decrease, respectively, in the NOCsupplemented group, compared with those of HF group (Figure 3A). PPAR- $\alpha, C P T-1 \alpha$, and UCP2 mRNA levels in WAT were analyzed to compare the effects of OC and NOC on $\beta$-oxidation and thermogenesis. The mRNA levels of PPAR- $\alpha, C P T-1 \alpha$, and UCP2 showed 2.92-, 3.8-, and 4.0-fold increase, respectively, in the OC group and 4.01-, 5.19-, and 5.76-fold increase, respectively, in the NOC group, compared with the HF group (Figure 3B). Moreover, the NOCsupplemented group showed higher expression of PPAR- $\alpha$, $C P T-1 \alpha$, and UCP2 mRNA levels by 1.36-, 1.52-, and 1.73fold, respectively, compared to the OC-supplemented group (Figure 3B).

\section{AMPK activity}

Effects of the OC and NOC on AMPK activity was determined in WAT, since AMPK activation affects mRNA expression of genes involved in adipogenesis and thermogenesis. After OC and NOC supplementation, AMPK activity was increased by $1.66-$ and 2.36 -fold, respectively, compared with the HF group $(P<0.01)$. In particular, the activity in the NOCsupplemented group was significantly higher than that in the OC-supplemented group $(P<0.01)$ (Figure 4A).

\section{GPDH activity}

We measured GPDH activity in WAT in view of the role of GPDH in adipogenesis. NOC supplementation inhibited GPDH activity by 1.34 -fold in comparison to that of the HF group $(P<0.05)$, whereas $\mathrm{OC}$ did not result in significant change (Figure 4B).

\section{Discussion}

A recent study has shown that red pepper constituents are associated with increased energy expenditure and thermogenesis, ${ }^{18}$ resulting in an anti-obesity effect. OC, alcoholic extract of red pepper, is lipophilic and poorly soluble, which means that making use of its effects is a challenging task. Therefore, NOC 

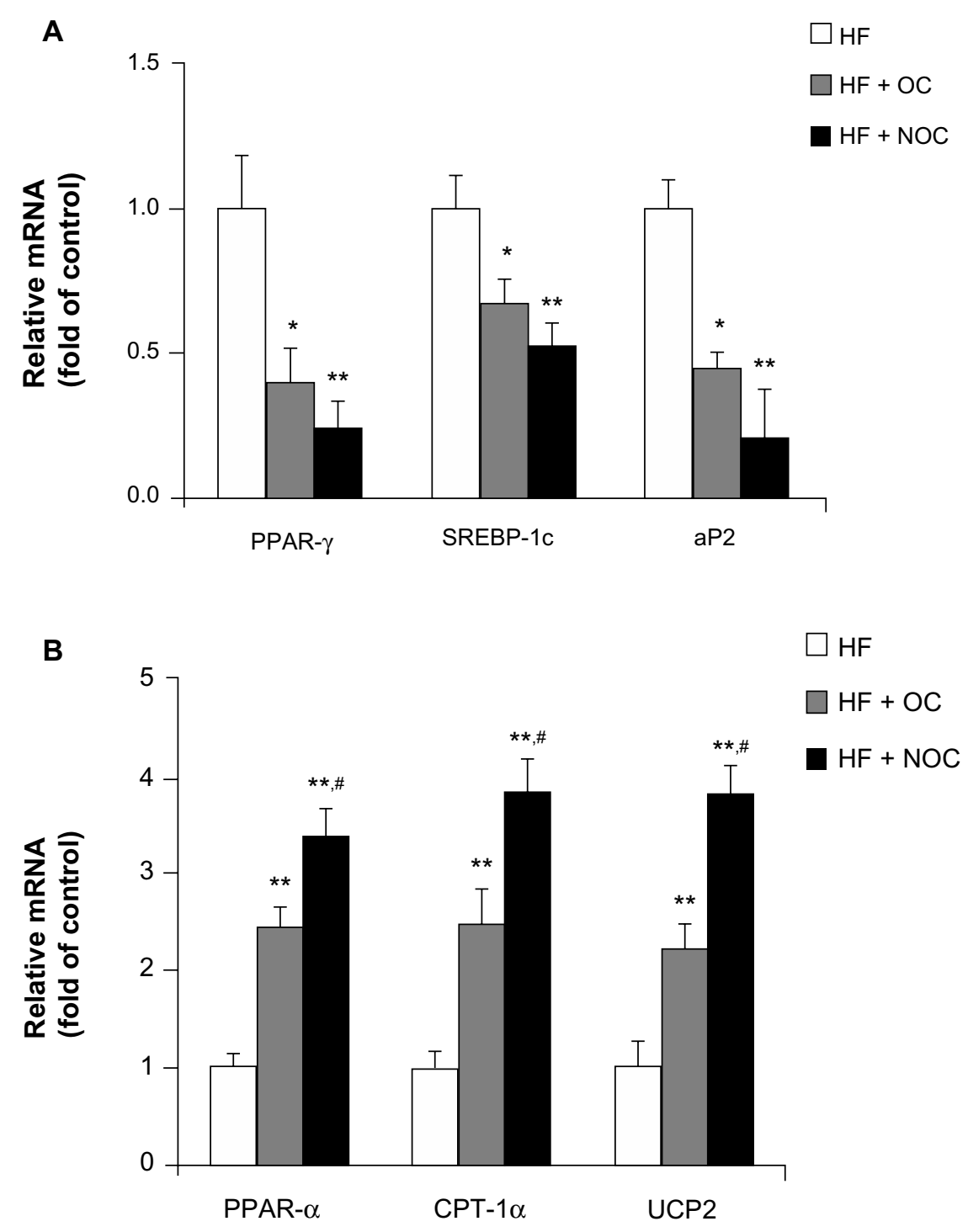

Figure 3 Effects of OC and NOC on mRNA expression of genes involved in energy metabolism in WAT.

Notes: Relative changes in mRNA levels for genes involved in (A) adipogenesis, such as PPAR- $\gamma$, SREBP-Ic, and aP2, and in (B) $\beta$-oxidation and thermogenesis, such as PPAR- $\alpha, C P T-I \alpha$, and UCP2. Total RNA samples were isolated from different treatment groups and analyzed by real-time PCR, and normalized to $\beta$-actin. The value of each control was set at the HF group value and was used to calculate the fold change in each target gene $(\mathrm{n}=7)$. $* P<0.05 ; * * P<0.01$ versus $\mathrm{HF}$ group; ${ }^{*} \mathrm{P}<0.05$ versus $\mathrm{HF}+\mathrm{OC}$ group.

Abbreviations: aP2, fatty acid-binding protein 2; CPT, carnitine palmitoyltransferase; HF, high-fat; NOC, nanoemulsion oleoresin capsicum; OC, oleoresin capsicum; PPAR, peroxisome proliferator-activated receptor; SREBP, sterol regulatory element-binding protein; UCP2, uncoupling protein-2; WAT, white adipose tissue.

was developed for improved bioefficacy of OC via enhanced bioavailability of lipophilic OC. The aim of this study was to examine the effects of $\mathrm{OC}$ and NOC on obesity, in particular, to assess whether they affect body weight and lipid profiles as well as enzyme activities and gene expression involved in energy metabolism.

Initial experiments were conducted to evaluate whether OC and NOC affected hepatotoxicity. The dose of OC and NOC used in our study was well tolerated by the rats, as demonstrated by the result that plasma levels of AST and ALT were not affected by OC and NOC supplementation. Body weight of the NOC-supplemented group was decreased significantly from 6 weeks after the beginning of the NOC-supplemented diet. The mass of WAT in the NOCsupplemented group was reduced compared to that in the HF group. Energy intake and energy efficiency ratio did not differ among the three groups. These results suggest that the reduction in body weight was not associated with an anorectic effect of the NOC. The OC- and NOC-supplemented groups showed reduction in the size of lipid droplets in adipocytes. WAT stores excess energy as TGs under conditions of energy excess. ${ }^{19}$ Increase in the size and number of adipocytes is a key feature of obesity. Thus, reduction in body weight, which could be attributed partially to a decrease in the mass of the fat 


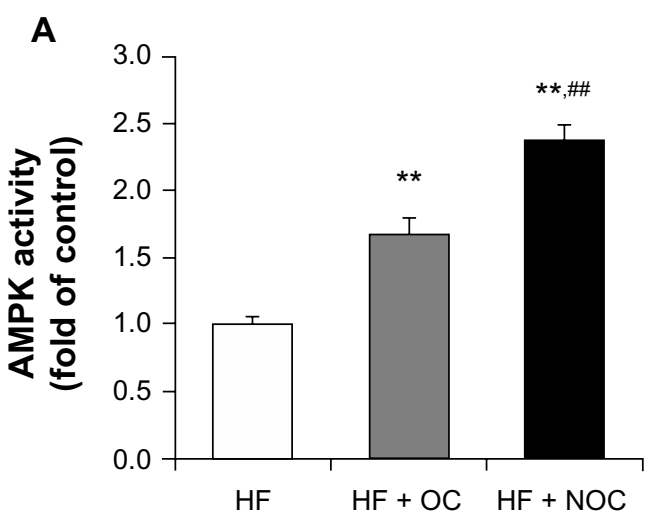

B

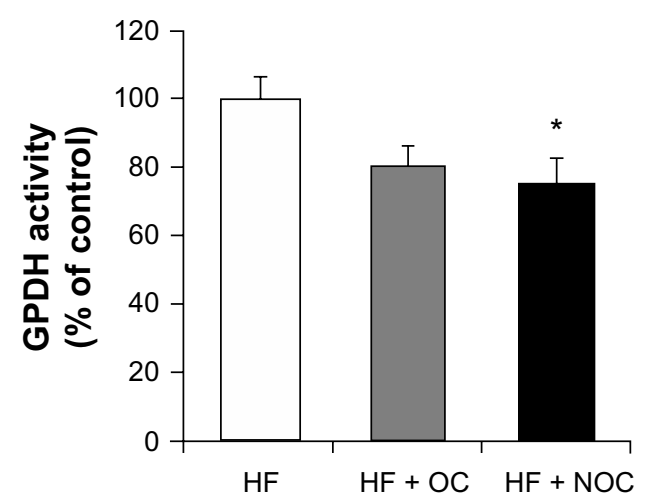

Figure 4 Effects of $O C$ and NOC on activity of enzymes involved in energy metabolism in WAT.

Notes: (A) AMPK activity; (B) GPDH activity. AMPK activity was measured using an AMPK kinase kit and normalized to protein content. The activity was expressed as fold increase of the HF group $(n=7)$. GPDH activity was measured using a GPDH kinase kit and normalized to protein content. Values for GPDH activity were expressed as a percentage of the HF group $(n=7)$. $* P<0.05$; $* * P<0.0$ I versus $\mathrm{HF}$ group; ${ }^{\prime} P<0.01$ versus $\mathrm{HF}+\mathrm{OC}$ group.

Abbreviations: AMPK, AMP-activated protein kinase; GPDH, glycerol-3phosphate dehydrogenase; HF, high-fat; NOC, nanoemulsion oleoresin capsicum; OC, oleoresin capsicum; WAT, white adipose tissue.

pad and size of adipocytes in the NOC-supplemented group, reflected a marked anti-obesity effect of NOC. The final body weight and adipose tissue mass of the OC-supplemented group tended to be lower than those in the HF group, but the difference was not statistically different. These results suggest that NOC efficiently prevented obesity.

Nanoemulsion technology is being increasingly utilized in the food industry to improve stability ${ }^{20}$ and oral bioavailability of lipophilic functional food components. ${ }^{21}$ As an example, the enhanced anti-inflammation activity of curcumin in $79.5 \mathrm{~nm}$ nanoemulsion droplets has been reported when compared with curcumin in $618.6 \mathrm{~nm}$ emulsion droplets in mice. ${ }^{22}$ In addition, supplementation with nano-sized dietary fiber significantly decreases plasma TG levels in comparison with control mice, whereas other forms of dietary fiber do not. ${ }^{23}$ In a previous study, in vivo pharmacokinetics analysis confirmed that $\mathrm{OC}$ nanoemulsions fabricated with chitosan/alginate polymers significantly enhanced the oral bioavailability of OC. ${ }^{24}$ In our experiment, it can be assumed, therefore, that improved bioavailability by using nanoemulsion processing might be one of the ways to explain the greater efficacy of NOC with reduced body weight and suppressed fat accumulation when compared with OC.

Various reports suggest beneficial effects of capsaicin in red pepper on plasma lipid parameters, although some reports question its therapeutic use. ${ }^{18,25}$ We have demonstrated herein that NOC effectively reduced the plasma level of TG. There were no differences in lipid concentration in the feces among the three groups. These data imply that reduction of body weight gain and plasma TG level in the NOC group might not result from the increase of TG excretion through lower intestinal TG absorption.

The mRNA levels of genes related to adipogenesis were analyzed in WAT to elucidate the anti-obesity action of OC and NOC. Adipogenesis is the process whereby preadipocytes become mature adipocytes. The rate of adipogenesis is crucial during expansion of WAT and, consequently, development of obesity. ${ }^{26}$ Several transcription factors, including PPAR- $\gamma$ and SREBP-1c, control the differentiation of preadipocytes into adipocytes, ${ }^{27}$ which is followed by increased adipogenesis in WAT. aP2, a carrier protein for fatty acids, is also associated with lipid biosynthesis pathways. ${ }^{28}$ In the present study, both OC and NOC significantly downregulated $P P A R-\gamma, S R E B P-1 c$, and $a P 2$ mRNA expression. These results suggest that the $\mathrm{OC}$ and NOC regulated fat accumulation by downregulating the expression of genes associated with adipogenesis and adipocyte differentiation.

Metabolic process that acts favorably on fatty acid oxidation in WAT is accompanied by upregulation of PPAR- $\alpha$ expression. $.^{29} C P T-1$ is an enzyme that increases fatty acid oxidation by controlling transfer of long-chain fatty acylcoenzyme A into the mitochondria. ${ }^{30}$ In our results, PPAR- $\alpha$ and $C P T-1 \alpha$ mRNA levels were increased following OC and NOC treatment. Moreover, NOC enhanced PPAR- $\alpha$ and $C P T-1 \alpha$ mRNA levels more efficiently than OC. UCP2 is expressed in various tissues, including brown adipose tissue, liver, WAT, skeletal muscle, kidney, and lung. Although UCP2 is not mainly responsible for thermogenic effect, the protein plays a key role in energy metabolism by functioning in adaptive thermogenesis, specifically when $U C P 2$ is activated fully by endogenous or exogenous environmental effectors. ${ }^{31,32}$ Considering that red pepper has an effect on stimulating heat generation, ${ }^{33}$ the increased UCP 2 mRNA level might contribute to the anti-obesity effect of the pepper. In our data, both 
OC and NOC significantly increased UCP2 mRNA levels. In addition, NOC supplementation showed higher expression of UCP 2 mRNA level than did OC supplementation. Therefore, it is suggested that increased fatty acid oxidation and energy expenditure as demonstrated by PPAR- $\alpha$ and UCP 2 levels might explain the relationship between NOC and its anti-obesity effects.

AMPK is an enzyme that regulates energy metabolism by stimulating catabolic pathways. In response to a decrease in the cellular adenosine triphosphate (ATP)/adenosine monophosphate (AMP) ratio, AMPK becomes activated through phosphorylation of its $\alpha$-subunit. ${ }^{34}$ Activated AMPK inhibits lipid biosynthetic pathways through downregulation of adipogenic genes such as SREBP-1C and PPAR- $\gamma$ and stimulates fatty acid oxidation through upregulation of the expression of PPAR- $\alpha$ and $P G C-1 \alpha .{ }^{34}$ AMPK activation has also been reported to increase the expression of $U C P 2$ in skeletal muscle, ${ }^{35}$ suggesting a role of AMPK activation for thermogenesis in the regulation of storage of body fat. In the present study, OC and NOC increased AMPK activity in WAT; moreover, NOC showed more stimulatory effect than OC. In our experiments, the activation of AMPK might have been related to the decreased levels of SREBP-1c and PPAR- $\gamma$ mRNA and increased levels of PPAR- $\alpha$ and UCP2 in the WAT of rats treated with NOC.

GPDH regulates energy metabolism towards the stimulation of lipogenesis by converting dihydroxyacetone phosphate to sn-glycerol-3-phosphate. ${ }^{36}$ In 3T3-L1 adipocytes, red pepper constituents decrease the amount of intracellular TG by inhibiting GPDH activity, which inhibits adipogenesis. ${ }^{37}$ In a previous study, one red pepper constituent, capsaicin, also attenuated GPDH activity, resulting in reduced WAT mass and body weight. ${ }^{36}$ Consistent with those results, NOC supplementation, in the present study, also led to decreased GPDH activity in WAT, whereas OC did not. From these results, it can be hypothesized that NOC is more effective at inhibiting GPDH activity than OC, and that inactivated GPDH may be partially related to anti-obesity effects of NOC.

\section{Conclusion}

In the present study, dietary NOC supplementation significantly reduced final body weight with a decreased mass of adipose tissue and ameliorated plasma TG levels in rats with obesity induced by a HF diet. However, the final body weight and WAT mass in the OC-supplemented group was not significantly different compared to those of the HF group. These effects were associated with downregulation of adipogenic gene expression, together with upregulation of gene expression of PPAR- $\alpha, C P T-1 \alpha$, and $U C P 2$, which are involved in $\beta$-oxidation and thermogenesis. It is likely that these anti-obesity effects of OC and NOC result from their ability to activate AMPK. In particular, the AMPK activity in the NOC-supplemented group was more stimulated than that of the OC-supplemented group. GPDH activity was more inhibited in the NOC group than the HF group. Meanwhile, there was no significant difference in the activity between the OC and HF group. We assume from these results that NOC might have obesity-preventing effects, which was associated with regulation of the expression of multiple genes, activation of AMPK, and inhibition of GPDH in rats fed a HF diet. Thus, NOC could serve as a promising agent for treatment of obesity.

\section{Acknowledgments}

This study was supported by the development of Food Nanotechnology, Korea Food Research Institute (No 20200703-03-WT011) and by a National Research Foundation of Korea (NRF) grant funded by the Korean Government (MOE) (No 2013R1A1A2009522).

\section{Disclosure}

The authors report no conflicts of interest in this work.

\section{References}

1. Hill JO, Peters JC. Environmental contributions to the obesity epidemic. Science. 1998;280(5368):1371-1374.

2. Magrone T, Perez de Heredia F, Jirillo E, et al. Functional foods and nutraceuticals as therapeutic tools for the treatment of diet-related diseases. Can J Physiol Pharmacol. 2013;91(6):387-396.

3. Haas JS, Whipple RE, Grant PM, Andresen BD, Volpe AM, Pelkey GE. Chemical and elemental comparison of two formulations of oleoresin capsicum. Sci Justice. 1997;37(1):15-24.

4. Dwivedi V, Shrivastava R, Hussain S, Ganguly C, Bharadwaj M. Cytotoxic potential of Indian spices (extracts) against esophageal squamous carcinoma cells. Asian Pac J Cancer Prev. 2011;12(8):2069-2073.

5. Zimmer AR, Leonardi B, Miron D, Schapoval E, Oliveira JR, Gosmann G. Antioxidant and anti-inflammatory properties of Capsicum baccatum: from traditional use to scientific approach. J Ethnopharmacol. 2012;139(1):228-233.

6. Jeon G, Choi Y, Lee SM, Kim Y, Jeong HS, Lee J. Anti-obesity activity of methanol extract from hot pepper (Capsicum annuum L.) seeds in 3T3-L1 adipocyte. Food Sci Biotechnol. 2010;19(4):1123-1127.

7. Olajos EJ, Salem H. Riot control agents: pharmacology, toxicology, biochemistry and chemistry. J Appl Toxicol. 2001;21(5):355-391.

8. Choi AJ, Kim CJ, Cho YJ, Hwang JK, Kim CT. Characterization of capsaicin-loaded nanoemulsions stabilized with alginate and chitosan by self-assembly. Food and Bioprocess Technology. 2011;4(6): 1119-1126.

9. McClements DJ, Rao J. Food-grade nanoemulsions: formulation, fabrication, properties, performance, biological fate, and potential toxicity. Crit Rev Food Sci Nutr. 2011;51(4):285-330.

10. Huang Q, Yu H, Ru Q. Bioavailability and delivery of nutraceuticals using nanotechnology. J Food Sci. 2010;75(1):R50-R57. 
11. Sagalowicz L, Leser ME. Delivery systems for liquid food products. Curr Opin Colloid Interface Sci. 2010;15(1-2):61-72.

12. Davis LT, Kumar N, Nijm LM, et al. An adaptable HPLC method for the analysis of frequently used antibiotics in ocular samples. J Chromatogr B Analyt Technol Biomed Life Sci. 2010;878(26):2421-2426.

13. Reeves PG. Components of the AIN-93 diets as improvements in the AIN-76 A diet. J Nutr. 1997;127 Suppl 5:S838-S841.

14. Bligh EG, Dyer WJ. A rapid method of total lipid extraction and purification. Can J Biochem Physiol. 1959;37(8):911-917.

15. Rozen S, Skaletsky H. Primer3 on the WWW for general users and for biologist programmers. Methods Mol Biol. 2000;132:365-386.

16. Livak KJ, Schmittgent TD. Analysis of relative gene expression data using real-time quantitative PCR and the $2^{-\Delta \Delta \mathrm{Ct}}$ method. Methods. 2001;25(4):402-408.

17. Lee MS, Kim IH, Kim CT, Kim Y. Reduction of body weight by dietary garlic is associated with an increase in uncoupling protein mRNA expression and activation of AMP-activated protein kinase in dietinduced obese mice. J Nutr. 2011;141(11):1947-1953.

18. Lee MS, Kim CT, Kim IH, Kim Y. Effects of capsaicin on lipid catabolism in 3T3-L1 adipocytes. Phytother Res. 2011;25(6):935-939.

19. Ahima RS, Flier JS. Adipose tissue as an endocrine organ. Trends Endocrinal Met. 2000;11(8):327-332.

20. Wang L, Li X, Zhang G, Dong J, Eastoe J. Oil-in-water nanoemulsions for pesticide formations. J Colloid Interface Sci. 2007;314(1): 230-235.

21. Kesisoglou F, Panmai S, Wu YH. Application of nanoparticles in oral delivery of immediate release formulations. Curr Nanosci. 2007;3(2): $183-190$.

22. Wang X, Jiang Y, Wang YW, Huang MT, Ho CT, Huang Q. Enhancing anti-inflammation activity of curcumin through $\mathrm{O} / \mathrm{W}$ nanoemulsions. Food Chem. 2008;108(2):419-424.

23. Wang ZQ, Yu Y, Zhang XH, et al. Comparing the effects of nano-sized sugarcane fiber with cellulose and psyllium on hepatic cellular signaling in mice. Int J Nanomedicine. 2012;7:2999-3012.

24. Choi AY, Kim CT, Park HY, et al. Pharmacokinetic characteristics of capsaicin-loaded nanoemulsions fabricated with alginate and chitosan. J Agric Food Chem. 2013;61(9):2096-2102.

25. Manjunatha H, Srinivasan K. Hypolipidemic and antioxidant effects of curcumin and capsaicin in high-fat-fed rats. Can J Physiol Pharmacol. 2007;85(6):588-596.
26. Gregoire FM, Smas CM, Sul HS. Understanding adipocyte differentiation. Physiol Rev. 1998;78(3):783-809.

27. Kim JB, Spiegelman BM. ADD1/SREBP1 promotes adipocyte differentiation and gene expression linked to fatty acid metabolism. Genes Dev. 1996;10(9):1096-1107.

28. Duplus E, Forest C. Is there a single mechanism for fatty acid regulation of gene transcription? Biochem Pharmacol. 2002;64(5-6):893-901.

29. Peters JM, Aoyama T, Burns AM, Gonzalez FJ. Bezafibrate is a dual ligand for PPARalpha and PPARbeta: studies using null mice. Biochim Biophys Acta. 2003;1632(1-3):80-89.

30. Lee WJ, Kim M, Park HS, et al. AMPK activation increases fatty acid oxidation in skeletal muscle by activating PPAR alpha and PGC-1. Biochem Biophys Res Commun. 2006;340(1):291-295.

31. Echtay KS, Roussel D, St-Pierre J, et al. Superoxide activates mitochondrial uncoupling proteins. Nature. 2002;415(6867):96-99.

32. Brand MD, Esteves TC. Physiological functions of the mitochondrial uncoupling proteins UCP2 and UCP3. Cell Metab. 2005;2(2):85-93.

33. Ludy MJ, Mattes RD. The effects of hedonically acceptable red pepper doses on thermogenesis and appetite. Physiol Behav. 2011;102(3-4): 251-258.

34. Rossmeisl M, Flachs P, Brauner P, et al. Role of energy charge and AMP-activated protein kinase in adipocytes in the control of body fat stores. Int J Obes Relat Metab Disord. 2004;28 Suppl 4:S38-S44.

35. Putman CT, Kiricsi M, Pearcey J, et al. AMPK activation increases uncoupling protein-3 expression and mitochondrial enzyme activities in rat muscle without fibre type transitions. $J$ Physiol. 2003;551(Pt 1): 169-178.

36. Ann JY, Lee MS, Joo H, Kim CT, Kim Y. Reduction of body weight by capsaicin is associated with inhibition of glycerol-3-phosphate dehydrogenase activity and stimulation of uncoupling protein 2 mRNA expression in diet-induced obese rats. J Food Sci Nutr. 2011;16(3): 210-216.

37. Hsu CL, Yen GC. Effects of capsaicin on induction of apoptosis and inhibition of adipogenesis in 3T3-L1 cells. J Agric Food Chem. 2007;55(5):1730-1736.
International Journal of Nanomedicine

\section{Publish your work in this journal}

The International Journal of Nanomedicine is an international, peerreviewed journal focusing on the application of nanotechnology in diagnostics, therapeutics, and drug delivery systems throughout the biomedical field. This journal is indexed on PubMed Central, MedLine, CAS, SciSearch $\AA$, Current Contents ${ }^{\circledR} /$ Clinical Medicine,

\section{Dovepress}

Journal Citation Reports/Science Edition, EMBase, Scopus and the Elsevier Bibliographic databases. The manuscript management system is completely online and includes a very quick and fair peer-review system, which is all easy to use. Visit http://www.dovepress.com/ testimonials.php to read real quotes from published authors. 